\title{
Percepción del envejecimiento exitoso en docentes de una facultad de medicina
}

\section{Perception of successful aging in faculty of a school of medicine}

\author{
Martha Martina ${ }^{1, a}$, Isabel Amemiya ${ }^{1, b}$, Julia Piscoya ${ }^{1, c}$, Héctor Pereyra $^{1, d}$, Zoila Moreno ${ }^{1, e}$ \\ ${ }^{1}$ Departamento Académico de Medicina Preventiva y Salud Pública, Facultad de Medicina, Universidad Nacional Mayor de San Marcos. Lima, Perú. \\ ${ }^{a}$ Doctora en ciencias de la salud, ORCID: https://orcid.org/0000-0002-8410-9120 \\ ${ }^{\mathrm{b}}$ Magister en medicina, ORCID: https://orcid.org/0000-0002-5502-4785 \\ ${ }^{\circ}$ Magister en epidemiologia, ORCID: https://orcid.org/0000-0002-4048-1326 \\ ${ }^{d}$ Magister en salud pública, ORCID: https://orcid.org/0000-0003-2449-4407 \\ ${ }^{e}$ Magister en docencia e investigación, ORCID: https://orcid.org/0000-0001-6071-5241
}

\section{An Fac med. 2019;80(2):167-72 / DOI: https:// 10.15381/anales.802.16411}

\section{Correspondencia: \\ Martha Martina Chávez}

mmartinac@unmsm.edu.pe

Recibido: 30 de abril 2019

Aceptado: 12 de junio 2019

Publicación en línea: 28 de junio 2019

Conflictos de interés: Los autores declaran no tener conflictos de interés

Fuente de financiamiento:

Autofinanciado

Citar como: Martina M, Amemiya I, Piscoya J, Pereyra H, Moreno Z. Percepción del envejecimiento exitoso en docentes de una facultad de medicina. An Fac med. 2019; 80(2):167-72. DOI: https:// 10.15381/ anales.802.16411

\section{Resumen}

Introducción. En las últimas décadas, en nuestro país se están produciendo cambios en la estructura poblacional por edad, uno de los más importantes es el incremento de la población adulta mayor. Objetivos. Determinar la percepción de vivir un envejecimiento exitoso e identificar algunas variables asociadas con dicha percepción en los docentes adultos mayores de la Facultad de Medicina de la Universidad Nacional Mayor de San Marcos. Métodos. Estudio transversal y correlacional. Muestra de 106 docentes adultos mayores seleccionados por conveniencia, entre septiembre a noviembre 2017. Se utilizó el Inventario de Envejecimiento Exitoso de Meredith Troutman proporcionada por la propia autora. Resultados. La edad media fue de 67,38 $\pm 5,77$ con un rango de 60 a 87 años. El $67 \%$ estaban casados; $47,7 \%$ vivía con su pareja y familiares, sólo cuatro docentes (3,8\%) vivian solos sin ningún apoyo. El 70,1\%(74) labora en la universidad 26 o más años; el 46,7\%(50) son asociados; el 64,2\%(68) es a tiempo parcial; el 46,7\%(49) tiene maestría y el $26,4 \%$ (28) pertenecen a la ONP Ley 20530. El 76,3\% de los docentes adultos mayores declararon que perciben un envejecimiento exitoso. Las dimensiones que más aportan al envejecimiento exitoso fueron mecanismos funcionales de rendimiento $(88,7 \%)$ seguido de satisfacción con la vida $(71,7 \%)$ y de los factores intrapsíquicos (69,5\%). Conclusiones. El envejecimiento exitoso es mayoritario en los docentes adultos mayores de la facultad de medicina probablemente como consecuencia de un proceso de adaptación a los cambios propios del envejecimiento y a una intensa actividad intelectual previa a esta etapa.

Palabras clave: Envejecimiento; Adulto Mayor; Docentes; Facultad de Medicina

\section{Abstract}

Introduction. In our country changes are taking place in the structure by age in the last decades, one of the most important is the increase of the older adult population. Objectives. To determine the perception of living a successful aging and identify some variables associated with this perception in the elderly teachers of the Faculty of Medicine, Universidad Nacional Mayor de San Marcos. Methods. Cross-sectional and correlational study. Sample of 106 older adult teachers selected for convenience, from september to november 2017. The Successful Aging Inventory of Meredith Troutman was used by the author. Results. The mean age was 67,38 \pm 5,77 with a range between 60 to 87 years. $67 \%$ were married; $47,7 \%$ lived with their partner and relatives, only four teachers $(3,8 \%)$ lived alone without any support. 70,1\% (74) work in the university for 26 years or more; $46,7 \%$ (50) are associates; $64,2 \%$ (68) work as part time; 46,7\% (49) have a master's degree and $26,4 \%(28)$ belong to the ONP Law 20530. 76,3\% of the elderly teachers declared that they perceive a successful aging. The dimensions that contribute most to successful aging were functional mechanisms of performance $(88,7 \%)$ followed by satisfaction with life $(71,7 \%)$ and intrapsychic factors $(69,5 \%)$. Conclusions. Successful aging is a majority in the elderly teachers of the faculty of medicine, probably as a result of a process of adaptation to the changes of aging and to an intense intellectual prior activity to this stage.

Keywords: Aging; Aged, Faculty; School, Medical 


\section{INTRODUCCIÓN}

El envejecimiento es una constante de la vida, pero adquiere relevancia en salud pública por los cambios demográficos que implica. No existe época en la historia en la que haya existido una composición demográfica con tantas personas mayores. Se interpreta este fenómeno como un éxito de las políticas de salud pública y consecuencia del desarrollo socioeconómico de los países ${ }^{1}$, pero al mismo tiempo significa un incremento de costos sanitarios, deficiente calidad de vida y carga familiar. Diversos consensos de asambleas mundiales y regionales han señalado la importancia del envejecimiento en el desarrollo de las sociedades $2,3,4$.

La Organización Mundial de la Salud (OMS) a finales de los años 90, definió envejecimiento activo como un proceso de optimización de las oportunidades de salud, participación y seguridad con el fin de mejorar la calidad de vida de las personas a medida que envejecen ${ }^{5}$. Sin embargo, las investigaciones sobre los adultos mayores suelen tratar acerca de su condición de persona dependiente, víctima de violencia ${ }^{6}$, estigma ${ }^{7}$, baja calidad de vida ${ }^{8}$, y son escasos los estudios sobre el adulto mayor en plena actividad intelectual. En el Perú, en las últimas décadas, se han producido importantes cambios en la estructura por edad. La población adulta mayor pasó de 5,7\% en 1950 a 10,1\% en el año 2017. Al tercer trimestre del año 2018, el 41\% de los hogares del país tenía entre sus miembros al menos una persona de 60 y más años de edad ${ }^{9}$.

Uno de los conceptos que hoy en día destaca es el de envejecimiento exitoso ${ }^{10-13}$ entre las cuales destaca la de Flood"1, definido como un "concepto multidimensional que abarca, trasciende y supera la buena salud y que está compuesto por un amplio conjunto de factores bio-psicosociales". El concepto de Flood se fundamenta en la teoría de la gerotrascendencia de Lars Tornstam quien introdujo una nueva comprensión del envejecimiento, afirmando que el desarrollo humano es un proceso de por vida que continúa en la vejez y que, cuando está optimizado, termina en una nueva perspectiva. La teoría de la gerotranscendencia se centra en dos fenóme- nos: la persona de edad y el proceso de envejecimiento en $\mathrm{sí}^{14}$.

Entre las investigaciones que han desarrollado instrumentos para medir el envejecimiento activo (exitoso), está la de Nusselder ${ }^{15}$ y la de Fernández-Ballesteros García y col. ${ }^{16}$, que encontraron como variables predictoras de envejecimiento activo el poseer una salud subjetiva, no necesitar ayuda, alto estado mental y alta satisfacción con la vida. Por su parte, Mauricio Blanco Molina ${ }^{17}$ señaló que algunos aspectos predictivos clave para que se establezcan estilos de vida activos en la vejez son la ausencia de estados depresivos, la constante actividad intelectual, algún grado de actividad física, la satisfacción con las redes de apoyo sociales y emocionales, el contar con un funcionamiento cognitivo que les permita adaptarse a las demandas del medio en el que se desenvuelven y la presencia de altos puntajes en escalas de autoeficacia percibida. Por su parte, Depp y Jeste ${ }^{18}$ encontraron una falta de claridad que rodea el significado de envejecimiento exitoso; la búsqueda bibliográfica de estudios que incluían una definición operacionalizada de envejecimiento exitoso, le permitió identificar 28 estudios con 29 definiciones diferentes.

En este contexto, Troutman y col. ${ }^{19}$ han desarrollado un instrumento para evaluar el envejecimiento exitoso "Successful Aging Inventory" (Inventario de Envejecimiento Exitoso - SAI). El instrumento evalúa cinco áreas asociadas al proceso de envejecer con "éxito": a) Mecanismos funcionales de rendimiento, b) Factores intrapsíquicos, c) Gerotrascendencia, d) Satisfacción con la vida y e) Espiritualidad. Estos mecanismos pueden adaptarse de manera sincronizada o no; ello determinará que la persona pueda o no envejecer con éxito en los dominios físicos, cognitivos y sociales. Este instrumento ha sido validado por Gallardo-Peralta y col. ${ }^{20}$ en adultos mayores chilenos, concluyendo que es confiable y válido, y que es una alternativa eficaz y rigurosa para aplicarse en otras sociedades y contextos culturales.

El segmento de adultos mayores en el ámbito universitario es poco estudiado. Así, El-Kawas ${ }^{21}$ destaca el fuerte compromiso de los docentes mayores de 50 años con las actividades de enseñanza, e investigación, por lo que parecería que la producción no disminuye con el paso de los años. Por su parte Bland y Bergquist citado por Rodriguez y colaboradores ${ }^{22}$, exploraron los factores asociados con la vitalidad académica y hallaron que a partir de los 50 años, los profesores experimentan profundos cambios, redefiniendo sus valores, metas y prioridades, sin que disminuya necesariamente su productividad en las actividades de enseñanza, adquiriendo la capacidad de adaptarse a las transformaciones. Destacan las hallazgos de Bensusán y Ahumada ${ }^{23}$ quienes investigaron a 30 mil académicos de tiempo completo activos y tres mil pensionados de 36 instituciones de educación superior mexicanas, concluyendo que el envejecimiento no fue un problema porque el promedio de edad de los profesores fue 48 años y sólo el 4,1\% tuvo más de 65; y la de González-Brambila y Veloso 24 quienes encontraron una relación directa entre la edad y la productividad, la misma que alcanza su máximo a los 53 años y decrece a partir de los 57,3 años.

En nuestro país, la Ley Universitaria N 30220 determinó que la edad máxima para el ejercicio de la docencia en la universidad pública es setenta años ${ }^{25}$, la misma que fue modificada por la Ley N³0697 (75 años) y que pasada esta edad sólo podrían ejercer la docencia bajo la condición de docentes extraordinarios. Según estas disposiciones, el fin de la carrera del docente universitario queda determinado por un asunto de edad, sin merituarse si el docente afronta o no un envejecimiento exitoso, situación que motiva la presente investigación. En nuestro país no existe evidencia de investigaciones sobre envejecimiento exitoso en un grupo poblacional en plena actividad intelectual; es por ello que el objetivo de la presente investigación fue determinar la percepción de vivir un envejecimiento exitoso e identificar algunas variables asociadas con dicha percepción en los docentes adultos mayores de la Facultad de Medicina de la Universidad Nacional Mayor de San Marcos.

\section{MÉTODOS}

Se realizó un estudio transversal, correlacional. Los datos fueron recolecta- 
dos entre septiembre a noviembre del 2017. La muestra estuvo conformada por 106 docentes adultos mayores (60 y más años), seleccionados por conveniencia, conservando la representatividad de los docentes de los departamentos académicos de ciencias básicas y clínicas.

Se utilizó el Inventario de Envejecimiento Exitoso de Meredith Troutman, validado en población adulta mayor chilena ${ }^{19}$ y cuya versión fue proporcionada por la propia autora quien autorizó su uso. El instrumento consta de 20 ítems (con acento) mecanismos funcionales de rendimiento (2 ítems), factores intrapsíquicos (7 ítems), gerotrascendencia (6 ítems), satisfacción con la vida (3 ítems), espiritualidad (2 ítems), todos los cuales se miden a través de una escala tipo Likert: (0) totalmente en desacuerdo a (4) totalmente de acuerdo. La puntuación varía de 0 a 80 . Considerando que un puntaje superior indica la presencia de un envejecimiento exitoso ${ }^{19}$, el equipo de investigadores determinó como punto de corte el tercer cuartil y según la tabla de probabilidad de la distribución normal el valor para el cual $\operatorname{Pr}(\mathrm{x} \leq \mathrm{z})=0,75$ correspondió a 0,674 por lo que se estimó dicho punto de corte $\square+0,674^{*}$ DS .

Las variables seleccionadas se clasificaron en dos grupos, socios demográficos y académicos. Respecto a las primeras fueron la edad (60 a 70 años y mayores de 70 años), el sexo, el estado civil se categorizó en dos grupos (solteros - casados. y otros) y si vivían solos o no. Las variables académicas fueron categoría docente( principal, asociado o auxiliar), clase docente (tiempo completo - dedicación exclusiva, y los de tiempo parcial), grado académico máximo obtenido a la fecha (bachiller, maestría y doctorado) y finalmente régimen de pensiones al cual pertenece.

Este inventario se aplicó mediante un Cuestionario autodesarrollado previa explicación al docente y contando con su consentimiento informado. Se aplicó estadística descriptiva. Para la asociación de variables se empleó el test de chi cuadrado. Se empleó el software: SPSS 21,0 . Se tomaron en cuenta los aspectos éticos para la recolección de datos, espe- cialmente se protegió la identidad de los informantes.

\section{RESULTADOS}

En la muestra estudiada (106 docentes) el rango de edad fue de 60 a 87 años, la edad media 67,38 $\pm 5,77$, el mayor porcentaje correspondió al grupo de 60 y 69 años (35,8 \%). Respecto al estado civil el $67 \%$ eran casados; el $47,7 \%$ vivía con su pareja y familiares, sólo cuatro docentes $(3,8 \%)$ vivían solos sin ningún apoyo. En cuanto a las características académicas y profesionales, el 70,1\% (74) laboraba en la universidad 26 o más años; el 46,7\% (50) de los docentes eran asociados; el 64,2\% (68) eran a tiempo parcial; el $46,7 \%$ (49) tuvo maestría y el 26,4\% (28) perteneció a la ONP Ley 20530.

El $76,3 \%$ de los docentes mayores de la muestra estudiada declararon que perciben un envejecimiento exitoso. Según se muestra en la tabla 1 , destaca la dimensión mecanismos funcionales de rendimiento $(88,7 \%)$ seguido de satisfacción con la vida $(71,7 \%)$ y de los factores intrapsíquicos (69,5\%); mientras que la

Tabla 1. Dimensiones del envejecimiento exitoso en docentes de la Facultad de Medicina de la Medicina de la Universidad Nacional Mayor de San Marcos, 2017.

\begin{tabular}{lccccccc}
\multirow{2}{*}{ Dimensiones } & \multicolumn{2}{c}{ Envejecimiento } & & \multicolumn{2}{c}{ Total } & \multirow{2}{*}{ p $^{*}$} \\
Mecanismos funcionales de rendimiento & Exitoso & No exitoso & & $\mathbf{n}$ & $\%$ & \\
\hline Alto & 74 & & & & & 0,001 \\
\hline Bajo & 4 & 8 & & & & & \\
\hline Satisfacción con la vida & & & & & & \\
\hline Alto & 71 & 5 & 76 & $71,7 \%$ & 0,000 \\
\hline Bajo & 7 & 23 & 30 & $28,3 \%$ & \\
\hline
\end{tabular}

Factores intrapsiquicos

\begin{tabular}{lccccc}
\hline Alto & 66 & 7 & 73 & $69,5 \%$ & 0,000 \\
\hline Bajo & 12 & 20 & 32 & $30,5 \%$ \\
\hline Gerotrascendencia & & & & & \\
\hline Alto & 32 & 0 & 32 & $30,5 \%$ & 0,000 \\
\hline Bajo & 46 & 27 & 73 & $69,5 \%$ & \\
\hline Espiritualidad & & & & & \\
\hline Alto & 21 & 1 & 22 & $21,4 \%$ & 0,015 \\
\hline Bajo & 57 & 24 & 81 & $78,6 \%$ & \\
\hline
\end{tabular}

Elaboración propia $\mathrm{n}=106$

* Chi cuadrado dimensión que más influyó en aquellos que no logran un envejecimiento exitoso, fue la gerotrascendencia y un bajo contacto con la espiritualidad. Para todos los casos el nivel de significancia fue menor de 0,01 .

Respecto a la asociación de algunas variables sociodemográficas con la percepción de envejecimiento exitoso, se encontró una mayor frecuencia en el grupo de 60 a 70 años, más en los docentes varones, en los están casados y los que viven acompañados; sin embargo, para ninguna de estas variables la diferencia encontrada fue estadísticamente significativa. Igual situación se produjo con las características académicas. Estos hallazgos se muestran en las tablas 2 y 3.

\section{DISCUSIÓN}

El objetivo de esta investigación fue determinar la percepción de vivir un envejecimiento exitoso e identificar algunas variables asociadas con dicha percepción en docentes adultos mayores en actividad de la Facultad de Medicina de la UNMSM. La inexistencia de inves- 
Tabla 2. Características socio demográficas y envejecimiento exitoso en docentes adultos mayores de la Facultad de Medicina de la Medicina de la Universidad Nacional Mayor de San Marcos, 2017.

\begin{tabular}{|c|c|c|c|c|}
\hline \multirow{2}{*}{ Caraterísticas académicas } & \multirow{2}{*}{ Total } & \multicolumn{2}{|c|}{ Envejecimiento } & \multirow{2}{*}{$\mathbf{p}^{*}$} \\
\hline & & Exitoso & No exitoso & \\
\hline \multicolumn{5}{|l|}{ Categoría docente } \\
\hline Principal & 47 & 37 & 10 & 0,332 \\
\hline Asociado & 50 & 36 & 14 & \\
\hline Auxiliar & 9 & 5 & 4 & \\
\hline \multicolumn{5}{|l|}{ Clase docente } \\
\hline Dedicación exclusiva & 7 & 7 & 0 & 0,243 \\
\hline Tiempo completo & 31 & 23 & 8 & \\
\hline Tiempo parcial & 68 & 48 & 20 & \\
\hline \multicolumn{5}{|l|}{ Grado académico } \\
\hline Doctorado & 29 & 24 & 5 & 0,230 \\
\hline Maestría & 49 & 37 & 12 & \\
\hline Bachiller & 27 & 17 & 10 & \\
\hline \multicolumn{5}{|l|}{ Sistema de pensiones } \\
\hline Régimen ONP 20530 & 28 & 23 & 5 & 0,622 \\
\hline Régimen 19990 & 38 & 26 & 12 & \\
\hline Sistema privado & 31 & 22 & 9 & \\
\hline
\end{tabular}

tigaciones en una población similar no nos permite realizar comparaciones; sin embargo, encontramos la investigación de Gallardo-Peralta ${ }^{26}$ quien estudió la asociación entre envejecimiento exitoso y participación social en personas mayores chilenas empleando el mismo instrumento y comprobó la relevancia que tiene la participación social durante la vejez como recurso psicosocial.

En esta investigación se halló un importante porcentaje de docentes que

perciben un envejecimiento exitoso, el cual supera el setenta y cinco por ciento. Una primera aproximación nos podría conducir a atribuir esta condición a la actividad laboral de los docentes, con un continuo ejercicio intelectual, y la interacción con muchas personas como sus alumnos, con exigencias de carácter cognoscitivo que contribuiría a fortalecer las redes de participación social ${ }^{26}$. Esta apreciación coincide con Mauricio Blanco Molina ${ }^{17}$, quien señaló que una de las cla-

Tabla 3. Características profesionales/académicas y envejecimiento exitoso en docentes adultos mayores de la Facultad de Medicina de la Medicina de la Universidad Nacional Mayor de San Marcos, 2017.

\begin{tabular}{|c|c|c|c|c|}
\hline \multirow{2}{*}{ Caraterísticas sociodemográficas } & \multirow{2}{*}{ Total } & \multicolumn{2}{|c|}{ Envejecimiento } & \multirow{2}{*}{$\mathbf{p}^{*}$} \\
\hline & & Exitoso & No exitoso & \\
\hline \multicolumn{5}{|l|}{ Grupos de edad } \\
\hline 60 a 70 años & 76 & 57 & 19 & 0,599 \\
\hline 71 y más años & 30 & 21 & 9 & \\
\hline \multicolumn{5}{|l|}{ Sexo } \\
\hline Mujer & 38 & 7 & 31 & 0,163 \\
\hline Hombre & 68 & 21 & 47 & \\
\hline \multicolumn{5}{|l|}{ Estado civil } \\
\hline Soltero & 20 & 5 & 15 & 0,873 \\
\hline Casado y otros & 86 & 23 & 63 & \\
\hline \multicolumn{5}{|l|}{ Con quien vive } \\
\hline Vive solo & 24 & 8 & 16 & 0,400 \\
\hline Vive acompañado & 81 & 20 & 61 & \\
\hline
\end{tabular}

Elaboración propia $\mathrm{n}=106$

* Chi cuadrado ves predictivas para que se establezcan estilos de vida activos en la vejez es una constante actividad intelectual y el contar con un funcionamiento cognitivo, que les permita adaptarse a las demandas del medio en el que se desenvuelven.

Por otra parte, la diversidad de características socio demográficas de la muestra y la multidimensionalidad del instrumento utilizado que implica factores ajenos a los de la sola actividad intelectual de los integrantes de la muestra, también alejan la interpretación de la unicausalidad del envejecimiento exitoso. Como indica Flood ${ }^{11}$, el envejecimiento exitoso es una percepción individual multifactorial, y por tanto debe analizarse desde las características particulares de los individuos de la muestra, lo que implicaría una investigación adicional de carácter cualitativo. El envejecimiento es un proceso ante todo individual, que conjuga la edad cronológica, la edad biológica, la edad psicológica, la edad social y la edad funcional, que se conjugan en el envejecimiento individual ${ }^{27}$.

Es importante precisar que ninguna teoría explica totalmente todos los cambios del proceso de envejecimiento, el cual es complejo y variado, describiéndose como un efecto acumulativo de la interacción de muchas influencias a lo largo de la vida, la herencia, el ambiente, las influencias culturales, la dieta, el ejercicio, la diversión, las enfermedades y otros muchos factores. Todo lo cual hace impredecible cómo y cuándo será el envejecimiento de una persona ${ }^{28}$. Algunos autores señalan que el envejecimiento es un proceso que se inicia en el nacimiento, cuando culmina el proceso de óptima funcionalidad y es secundado por el deterioro de la misma y su involución ${ }^{27}$.

Una de las dimensiones que más contribuyó al envejecimiento exitoso en la muestra estudiada fue la referida a los mecanismos funcionales de rendimiento, representada por dos preguntas: ¿yo consigo hacer todas las cosas que necesito, como hacerme cargo de mi casa y de mi persona? y ¿he sido capaz de hacer frente a los cambios que han ocurrido a mi cuerpo conforme he envejecido? Esta dimensión evalúa la capacidad de conciencia y elección de la persona mayor como una respuesta adaptativa a las pérdidas fisiológicas y físicas producto 
del envejecimiento ${ }^{26}$, ello podría explicarse por el nivel intelectual del grupo de estudio, que le permite adaptarse a este nuevo período de su vida.

La dimensión satisfacción con la vida estuvo representada por tres preguntas: ¿mi vida tiene sentido?, ¿estoy en general satisfecho con mi vida ahora mismo? y ¿siento que sirvo a un propósito en este mundo?. Es la dimensión que alcanza los niveles más altos de una dimensión individual en aquellos que alcanza una puntuación de envejecimiento exitoso y es la dimensión individual con más bajo puntaje cuando no alcanzan un envejecimiento exitoso (71,7\% versus $28,3 \%$ ). Al ser la satisfacción con la vida una percepción individual, se puede interpretar la diferencia de valores entre los que desarrollan un envejecimiento exitoso y aquellos que no lo logran como consecuencia de diferentes expectativas y por tanto de diferentes niveles de adaptación a los cambios propios del envejecimiento.

La dimensión factores intrapsiquicos tuvo preguntas como: ¿me siento capaz de enfrentar mi propio envejecimiento?, ¿me siento capaz de hacer frente a los acontecimientos de la vida?, ¿puedo solucionar los problemas?, ¿puedo encontrar nuevas formas de resolver problemas?, ¿me gusta hacer cosas nuevas o creativas?, y ¿estoy en un estado de ánimo positivo y agradable?. Es la tercera dimensión en alcanzar el puntaje más alto en aquellos que alcanzan un envejecimiento exitoso e igualmente es la tercera dimensión con puntaje más bajo en aquellos que no desarrollan un envejecimiento exitoso. Estos factores corresponden a la flexibilidad y adaptabilidad para resolver los problemas de la vida diaria a través de la creatividad. Constituyen mecanismos de adaptación a los cambios de la edad, fomentan el optimismo y permiten el autocontrol.

La espiritualidad como dimensión presenta un resultado inverso. En aquellos que alcanzan un envejecimiento exitoso su valor es el más bajo de las dimensiones evaluadas, y al mismo tiempo en aquellos que no alcanzan el envejecimiento exitoso es el valor más alto. Esto implica que la espiritualidad por sí sola no contribuye significativamente a un envejecimiento exitoso en esta población.
San Martín ${ }^{28}$ plantea una diferencia entre religiosidad y espiritualidad, concibiendo al primero como de naturaleza social y comprende un cuerpo de conocimientos, ritos, normas y valores que rigen la vida del individuo interesado en vincularse con lo divino. Mientras que la naturaleza de la espiritualidad es singular, específica y personal; se caracteriza por un sentimiento de integración con la vida y con el mundo, y se vive como la experiencia de lo divino.

Podría deducirse de estos resultados que la espiritualidad toma forma de trascendencia cósmica, de aceptación del lugar y posición que tiene el individuo en un universo del cual forma parte y por tanto la aceptación del destino de la vida y la muerte como realidades que superan al individuo. La aceptación de esta perspectiva se vincula con una interpretación más que vinculada a fenómenos trascedentes como corresponde a la espiritualidad, a los fenómenos cósmicos propios de la gerotrascendencia.

En cuanto a la gerotrascendencia, las preguntas que definen esta dimensión estuvieron representadas por: ¿pienso en mis seres queridos que han fallecido y me siento cerca de ellos?, ¿como ya he envejecido, mi forma de pensar del mundo ha cambiado?, ¿prefiero tener unos pocos amigos íntimos que muchas ocasiones casuales?, ¿siento interés/preocupación por la próxima generación? ¿la vida con la edad que tengo ahora, es tan buena o mejor de lo que pensé que sería?. Al respecto, Lars Tornstam ${ }^{14}$ sociólogo sueco, describe gerotrascendencia como "un cambio desde una visión materialista y racional del mundo a una más cósmica y trascendente, normalmente acompañada de un aumento de la satisfacción con la vida".

En cuanto a variables asociadas al envejecimiento exitoso como los grupos etarios, género, estado civil, la convivencia o no con otras personas, la categoría y clase docente, el grado académico, y el régimen pensionario de los docentes, no se encontraron diferencias significativas desde el punto estadístico. No obstante, hemos encontrado envejecimiento exitoso mayor en el grupo de 60 a 70 años que los de 70 y más años (2:1); los hombres más que las mujeres (3:1); los que están casados respecto a los solteros $(4: 1)$ y los que viven acompañados (2:1).

Al analizar las características académicas, del total de los docentes a dedicación exclusiva (7) y un grupo mayoritario de docentes a tiempo completo (23/31) percibieron que viven un envejecimiento exitoso. Igualmente los que poseen grado de doctor y maestría, así como los docentes principales y asociados. Destacan también aquellos docentes que pertenecen al régimen de pensiones de la ONP 20530. Para este grupo de docentes la percepción de vivir un envejecimiento exitoso estaría explicado por experimentar una seguridad económica y satisfacción de altos logros académicos.

En conclusión, a percepción de envejecimiento exitoso en los docentes adultos mayores de la Facultad de Medicina UNMSM es alto (76,3\%). Las dimensiones que más contribuyeron a la percepción de un envejecimiento exitoso fueron los mecanismos funcionales de rendimiento y satisfacción con la vida. No se encontró asociación estadística entre envejecimiento exitoso y las variables seleccionadas. Es importante continuar con esta línea de investigación con el fin de superar algunas limitaciones de este estudio, como el haber seleccionado una muestra no probabilística, el no incluir co-morbilidades y sólo con docentes en actividad. Otro factor que consideramos como una limitación es la escasez de investigaciones que exploren esta temática en este grupo poblacional. Igualmente sugerimos abordar este tema con un enfoque cualitativo. No obstante las limitaciones expuestas, consideramos que este trabajo constituye un punto de partida para posteriores investigaciones.

\section{REFERENCIAS BIBLIOGRÁFICAS}

1. Naciones Unidas. Plan de Acción Internacional de Viena sobre el Envejecimiento. Asamblea Mundial Sobre el Envejecimiento. Viena, Austria, 26 julio a 6 de agosto de 1982

2. Naciones Unidas. Informe de la Segunda Asamblea Mundial sobre el Envejecimiento. In Madrid; 2002. p. 127.

3. Naciones Unidas. Declaración de Brasilia. Segunda Conferencia Regional Intergubernamental sobre envejecimiento en América Latina y el Caribe: hacia una sociedad para todas las edades y de protección social basa en derechos. Brasilia, 2007 
4. Naciones Unidas. Carta de San José sobre los derechos de las personas mayores de América Latina y el Caribe. San José de Costa Rica, 8 al 11 mayo; 2012

5. Organización Mundial de la Salud. Informe Mundial sobre el Envejecimiento y la Salud 2015

6. Silva-Fhon J, Del Rio-Suarez A, Motta-Herrera S, Fabricio-Wehbe S, Partezani-Rodrigues R. Violencia intrafamiliar en el adulto mayor que vive en el distrito de Breña, Perú. rev.fac. med. 2015 63(3):367-375. DOI: http://dx.doi. org/10.15446/revfacmed.v63n3.44743

7. Scazufca M, P de Paula Couto MC, Huang H, Kester R, Braga PE, Peluso ÉT, et al. Public Stigma towards Older Adults with Depression: Findings from the São Paulo-Manaus Elderly in Primary Care Study. PLoS One. 2016;11(6):e0157719. DOI: DOI: 10.1371/journal.pone.0157719

8. Shah VR, Christian DS, Prajapati AC, Patel MM Sonaliya KN. Quality of life among elderly population residing in urban field practice area of a tertiary care institute of Ahmedabad city, Gujarat. J Family Med Prim Care. 2017;6(1):101-105. DOI 10.4103/2249-4863.214965.

9. Instituto Nacional de Estadistica e Informática [Internet]. Situación de la Población Adulta Mayor. Inf Técnico N ${ }^{\circ} 42018$ [Fecha de acceso: 12 de mayo 2019]. Disponible en: https://www.inei.gob.pe/biblioteca-virtual/boletines/ninez-y-adulto-mayor/1

10. Rowe JW, Kahn RL. Human aging: usual and successful. Science. 1987; 237: 143-9.

11. Flood M. Successful aging: a concept analysis. J Theory Constr Test. 2002;6(2):105-8

12. Cosco TD, Prina AM, Perales J, Stephan BC Brayne C. Operational definitions of successful aging: a systematic review. Int Psychogeriatri. 2014; 26 (3): 373-81.

13. Cheng ST. Defining successful aging: the need to distinguish pathways from outcomes. Int Psychogeriatr. 2014;26(4):527-31.

14. Wadenstein B. The theory of gerotranscendence applied to gerontological nursing - Part I. Int J Older People Nurs. 2007;2(4):289-94. DOI: 10.1111/j.1748-3743.2007.00085

15. Nusselder WJ, Peeters A. Successful aging: measuring the years lived with functional loss. J Epidemiol Community Health. 2006;60(5):448-55. DOI: 10.1136/jech.2005.041558

16. Fernández-Ballesteros $R$, Caprara $M$, Iñiguez J. Promoción del envejecimiento activo: efectos del programa «Vivir con vitalidad». Rev esp. ger. 2005; 40(2):92-102.

17. Blanco M. Predictores psicosociales del envejecimiento activo: Evidencias en una muestra de personas adultas mayores. Anales en Gerontologia. 2010;(6):11-29.

18. Depp CA, Jeste DV. Definitions and predictors of successful aging: A comprehensive review of larger quantitative studies. Am J Geriatr Psychiatry. 2006;14(1):6-20. DOI: 10.1097/01. JGP.0000192501.03069.bc

19. Troutman M, Nies MA, Small S, Bates A. The Development and Testing of an Instrument to Measure Successful Aging. Res Gerontol Nurs. 2011; 4(3):221-32. DOI: 10.3928/19404921-20110106-02

20. Gallardo-Peralta L, Cuadra-Peralta A, Camara-Rojo X, Gaspar-Delpino B, Sánchez-Lillo R. Validación del inventario de envejecimiento exitoso en personas mayores chilenas. Rev. méd. Chilena. 2017; 145(2): 172-180. DOI: http://dx.doi.org/10.4067/S0034-98872017000200004
21. El-Khawas E. Senior Faculty in Academe: Active Committed to the Teaching Role. Res Briefs. 1991;2(5).

22. Rodriguez J, Urquidi L, Mendoza G. Edad, Producción Académica y Jubilación en la Universidad de Sonora. Rev Mex Investig Educ. 2009;14:593-617.

23. Bensunsán G, Ahumada Lobo İ. Sistemas de jubilación en las instituciones públicas de educación superior y composición por edad del personal académico. Rev la Educ Super. 2006;138(138):7-35.

24. Gonzalez-Brambila C, Veloso F. The Determinants of Research Productivity: A Study of Mexican Researchers. Research Policy. 2007;36(7):10351051. DOI: 10.1016/j.respol.2007.03.005

25. Ministerio de Educación del Perú [Internet]. Ley Universitaria $\mathrm{N}^{\circ} 30220$ [Fecha de acceso 12 de mayo 2019]. Disponible en: http://www.minedu gob.pe/reforma-universitaria/pdf/ley_universitaria.pdf

26. Gallardo-Peralta L, Conde-Llanes D, Córdova-Jorquera I. Asociación entre Envejecimiento exitoso y participación social en personas mayores chilenas. Gerokomos. 2016;27(3):104-108.

27. González Bernal, de la Fuente J. Desarrollo Humano en la Vejez: Un envejecimiento óptimo desde los cuatro componentes del ser humano. Internacional Journal of Developmental and Educational Psychology. 2014;7(1):12-129

28. Troen BR. The biology of aging. Mt Sinai J Med. 2003; 70(1):3-22

29. San Martín Petersen Cecilia. La espiritualidad en el proceso de envejecimiento del adulto mayor. Universidad de La Frontera, Temuco, Chile. Hologramática. Año V, Número 8, V1 (2008), 103-120 